\title{
Médecines parallèles : un révélateur de la relation médecin-malade
}

\author{
Alternative medicine: an indicator of the doctor-patient relationship
}

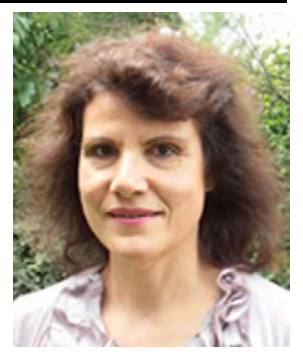

\author{
M.-F. Bacqué \\ (C) Springer-Verlag France 2011
}

\section{Mieux définir médecines alternatives et complémentaires}

Le vocable de médecines parallèles recouvre toutes les thérapeutiques utilisées par un patient en plus ou à la place des thérapeutiques conventionnelles. Il faut cependant distinguer deux types de traitements autoadministrés : d'une part, les médecines alternatives qui prennent la place des traitements conventionnels, d'autre part, les médecines complémentaires qui s'ajoutent aux thérapeutiques allopathiques pour améliorer l'état de santé du patient et ses chances de guérison.

Les médecines complémentaires ne doivent pas être confondues avec les médecines alternatives. Cependant, toutes soulignent les limites des traitements conventionnels et montrent l'intérêt du patient pour sa maladie, son désir d'être actif, sa référence à d'autres systèmes d'interprétation de la maladie et de la guérison.

\section{Qui utilise les médecines parallèles ?}

Si jadis, les malades du cancer se mettaient en quête de conseils de toute sorte et étaient susceptibles d'appliquer les thérapeutiques les plus désespérées en l'absence de connaissances rationnelles, aujourd'hui, ceux qui ont recours aux médecines complémentaires sont pragmatiques et plutôt bien informés. Des études de populations, aux États-Unis comme en France, montrent que les utilisateurs principaux de compléments médicaux sont des femmes, jeunes (de 30 à 50 ans), de niveau socioculturel élevé, et dans le cas d'un cancer du sein, qui ont une perception des risques de récidive et de leur mortalité plus élevée que les autres.

Mais, au fait, quel pourcentage de patients atteints de cancer emploie-t-il les médecines complémentaires et

\section{M.-F. Bacqué ( $\varangle)$}

Université de Strasbourg,

12, rue Goethe, F-67000 Strasbourg, France

e-mail : mfbacque@club-internet.fr alternatives ? Pour la France, on en trouverait $36 \%$, soit en fait quasiment autant qu'aux États-Unis.

Toutefois, à nouveau, il vaudrait mieux distinguer médecines alternatives et complémentaires. Le temps semble révolu où les patients « échappaient 》 totalement aux protocoles bien établis en oncologie. L'alternative ne semble plus de mise. Lorsqu'elle existe, il s'agit plutôt de refus de traitement. Les Refuseniks, dont le Time se faisait l'écho le 27 juin 2011 [3], sont ces patients plutôt âgés, qui, en toute connaissance de cause, décident de passer outre les chances de guérison (souvent faibles) pour éviter les effets secondaires des traitements et une fin de vie qu'ils imaginent à la solde de la médecine. Ces Refuseniks ont été retrouvés jusqu'à présent chez les femmes âgées de plus de 65 ans avec un cancer du sein et chez les hommes âgés qui craignent impuissance et incontinence, séquelles de la prostatectomie.

Peut-on mettre les Refuseniks à la base de l'axe qui s'orienterait du refus de soin vers l'utilisation de médecines complémentaires pour, à l'autre extrémité, trouver les malades en adhésion complète et unique avec l'allopathie ?

De nombreux chercheurs ont tenté de trouver une typologie de la " désobéissance médicale » et finalement montré que les patients utilisateurs de médecines alternatives ou complémentaires n'étaient ni plus anxieux ni plus déprimés que les autres. Par ailleurs, utiliser des médecines complémentaires n'empêche absolument pas de suivre son traitement. Les utilisateurs sélectifs de médecines alternatives sont donc rares, sauf peut-être chez les Refuseniks qui sortent définitivement de la filière médicale classique.

\section{L'absence de dialogue sur les médecines complémentaires limite la confiance médecin-malade}

Ce qui semble en revanche justifier un débat de fond parmi les médecins, et plus spécifiquement les oncologues, concerne la faiblesse de la communication autour des médecines complémentaires et alternatives. 
Pour les Refuseniks, la sortie d'un protocole de traitement anti-cancer ou le refus d'y entrer ne devrait pas entraîner une rupture de dialogue avec le médecin. Si l'on comprend le refus de s'investir d'un oncologue déjà chargé de nombreuses vies, le soutien du médecin généraliste devrait être accru. Or, l'enquête magistrale menée par J.-M. Dilhuidy en 2003 [2] pose le constat bien français de l'incroyable fossé qui sépare les patients qui, pour $75 \%$ d'entre eux, aimeraient partager avec leur cancérologue leurs questions ou leur choix de médecines alternatives ou complémentaires et la grande majorité des médecins ( $71 \%$ ) qui ne souhaitent pas en parler. Une telle différence ne montre-t-elle pas un problème majeur de communication à l'origine de possibles malentendus graves ?

Effectivement, lorsque S. Schraub et son équipe sondent leurs 46 patients français atteints de cancer sur les raisons qui les amènent à utiliser des médecines complémentaires, on observe que c'est la qualité de la relation avec le thérapeute qui est principalement recherchée. Les médecines parallèles ont été prescrites par un médecin conseillé par famille ou amis dans la quasi-majorité des cas, d'autre part, c'est la relation plus complète, prenant en compte le confort, la douleur, le besoin d'explication, qui satisfait le patient. Ce dernier ne délaisse pas les médecines conventionnelles pour autant, mais le médecin « complémentaire » est choisi pour son approche holistique, tandis que l'oncologue reste le spécialiste qui soutient la science et ses protocoles non personnalisés.

\section{Médecines parallèles ou l'Autre qui guérit}

J.-L. Bagot et son coauteur pensent que des questions de pouvoir sont aussi à l'œuvre dans cette frontière française entre médecine globale du sujet, y compris dans sa subjectivité et ses croyances, et médecine allopathique académique, égalitaire socialement certes, mais non distinctive subjectivement. Les connaissances actuelles des malades atteints de cancer, mais peut-être aussi une certaine forme de syncrétisme religieux, les conduisent à opter pour le maximum de chances, même si cela ne correspond pas à une approche culturelle homogène. On connaît l'attrait actuel des Français pour le bouddhisme qui serait devenu la cinquième « religion» (il s'agit en réalité d'une véritable philosophie) de notre pays. Si certains concoctent une forme de bricolage qui les « arrange », il n'est pas sûr qu'ils tirent tous les bénéfices de ces mélanges de symboles ou de signifiants qui résultent de l'évolution d'une histoire et de cultures propres à des peuples très éloignés de nous. On observe ainsi que le terme « énergie » est très présent dans la médecine chinoise. Il est en revanche totalement absent des médecines conventionnelles européennes. Les patients férus de philosophies orientales sont sans doute dans l'attente d'une telle défini- tion. La méditation en pleine conscience vient répondre à cette demande. «L'énergie mentale » qu'ils pourraient développer les séduit du côté de leur participation. Pour la plupart des malades jeunes, révoltés par leur cancer, la recherche d'une énergie interne latente les conforte devant une affection dont la fatalité les assomme.

\section{Médecines complémentaires d'aujourd'hui : un syncrétisme moderne}

B.R. Cassileth fait la synthèse de ces propos. La psychooncologie se veut intégrative. Qu'à cela ne tienne, le MS-KCC (Memorial Sloane-Kettering Cancer Center) propose un programme qui regroupe les avantages de toutes ces options en gardant sans doute le meilleur. On peut se demander s'il n'est pas provocateur de délaisser la philosophie d'une religion ou d'une culture pour n'en utiliser que les techniques. Mais de tout temps, les hommes ont domestiqué les dieux avec pragmatisme, la spiritualité étant réservée à une élite finalement. Nous voilà donc avec un bricolage à l'américaine, mais qui a le mérite de reconnaître ce qui marche !

Ainsi aux États-Unis, tous les services d'oncologie offrent au moins une thérapie complémentaire. Le bilan mené au MS-KCC pendant trois ans sur environ 1300 patients [1] a montré que les massages soulageaient la douleur, diminuaient la fatigue et les nausées et que ces bénéfices persistaient au moins 48 heures. Une étude de 2010 sur la pratique du yoga par 410 patients atteints de cancer (peu évolué) montre son amélioration de la qualité du sommeil, une diminution de la fatigue et, globalement, une augmentation de la qualité de vie [4].

L'acupuncture diminue de nombreux symptômes comme la dyspnée, la fatigue, les troubles du climatère, les dysfonctionnements sexuels. Enfin, la musicothérapie limite l'isolement du malade et fait participer familles et soignants à la prise en charge du patient en améliorant les échanges du groupe.

Il est à noter que le site www.mskcc.org/aboutHerbs offre des réponses claires sur les interactions avec les plantes et autres produits censés faire repousser les cheveux ou réduire les bouffées de chaleur. Mais principalement, la politique du MS-KCC est énoncée sans ambiguité : les médecines alternatives sont mises de côté, car elles n'ont pas fait leur preuve et éliminent dangereusement les thérapeutiques conventionnelles. Les médecines complémentaires validées sont en revanche largement mises en avant pour leur innocuité et leurs résultats favorables sur la qualité de vie des patients. Elles sont, de plus, remboursées par la plupart des assurances américaines. Enfin, toutes les questions concernant les régimes alimentaires, les pratiques sportives, mais aussi les 
compléments alimentaires dont les Américains sont friands, sont abordées avec franchise.

\section{Le succès des médecines complémentaires en France dans l'aura des gourous?}

À ce sujet, nous ne pouvons pas ne pas nous questionner sur le succès considérable des ouvrages de D. Servan-Schreiber mort le 24 juillet 2011 du cancer qui l'avait conduit à tester sur sa personne de nombreuses thérapies complémentaires. Il a sans doute permis d'ouvrir ce champ plus avant. Il est cependant dommage qu'une personne privée et singulière (mais médecin et chercheur) promeuve ce qu'oncologues et biologistes auraient aussi pu soutenir de façon plus collective et scientifiquement approuvée. Peut-être aura-t-il encore fallu que les Français soutiennent une approche de type « gourou » (ce dont DSS se défendait) pour aborder franchement les médecines complémentaires. Par ailleurs, ces médecines complémentaires qui apportent plus que du « confort», seront-elles un jour remboursées par la Sécurité sociale?

Les médecines alternatives doivent donc être clairement séparées des médecines complémentaires qui ont fait la preuve de leur amélioration de la qualité de vie des patients.
Aujourd'hui, les praticiens ne peuvent plus dénier leur utilité et le désir qu'ont les patients d'en parler. Elles font partie de l'humanisation des services d'oncologie pour lesquels les traitements « de pointe » ont conduit à mettre de côté l'approche subjective des patients. Avec la reconnaissance de ces apports thérapeutiques (encore trop discrets dans les soins de support), souhaitons qu'un dialogue se développe autour de projets thérapeutiques permettant définitivement une meilleure implication des patients.

\section{Références}

1. Cassileth BR, Vickers AJ (2004) Massage therapy for symptom control: outcome study at a major cancer center. J Pain Symptom Manage 28:244-9

2. Dilhuydi JM (2003) L'attrait pour les médecines complémentaires et alternatives en cancérologie : une réalité que les médecins ne peuvent ni ignorer, ni réfuter. Bull Cancer 90:623-8

3. Konigsberg RD (2011) The Refuseniks. Why some patients reject their doctor's advice. Time, June 27, 2011

4. Mustian KM, Palesh O, Sprod L, et al (2010) Effects of YOCAS yoga and sleep, fatigue and quality of life: a URCC CCOP randomized controlled clinical trial among 410 cancer survivors. J Clin Oncol 28:15s (suppl; abstr 9013) 